\title{
Differential microRNA expression profiles in tamoxifen-resistant human breast cancer cell lines induced by two methods
}

\author{
PENG YE* ${ }^{*}$, CHENG FANG $^{*}$, HUI ZENG ${ }^{*}$, YU SHI, ZHONGYA PAN, \\ NAIRUI AN, KELI HE, LI ZHANG and XINGHUA LONG \\ Department of Laboratory Medicine, Zhongnan Hospital of Wuhan University, Wuhan, Hubei 430071, P.R. China
}

Received March 7, 2017; Accepted November 20, 2017

DOI: $10.3892 / \mathrm{ol} .2018 .7768$

\begin{abstract}
Tamoxifen (TAM) resistance has become a severe problem for endocrine therapy of breast cancer. The present study investigated the association between microRNA (miRNA) expression and TAM resistance in breast cancer. The TAM-resistant breast cancer MCF-7C and MCF-7T cell lines were established using the human breast cancer cell line MCF-7 as the parental cell line and 4-hydroxytamoxifen (OHT) as the screening drug in vitro. The MCF-7C cell line was established by dose stepwise induction beginning with a low concentration of OHT; the MCF-7T cell line was established by temporal stepwise induction beginning with a high concentration of OHT. Differential miRNA expression profiles between TAM-sensitive (MCF-7) and TAM-resistant (MCF-7C and MCF-7T) breast cancer cell lines were detected and analyzed using RNA sequencing technology. The results of western blot analysis indicated that the level of ER $\alpha$ protein expression in drug-resistant cells was significantly increased. A total of 1,646 miRNAs were detected in all samples, including 1,376 known miRNAs and 270 predicted miRNAs. There were 118 miRNAs expressed at significantly different levels between MCF-7C and MCF-7 cells $(\mathrm{P}<0.05)$; among them, 67 miRNAs were upregulated $(\mathrm{P}<0.05)$ and 51 miRNA were downregulated $(\mathrm{P}<0.05)$. There were $42 \mathrm{miRNAs}$ expressed at significantly different levels between MCF-7T and MCF-7 $(\mathrm{P}<0.05)$; among them, 23 miRNAs were upregulated $(\mathrm{P}<0.05)$ and 19 miRNAs were downregulated $(\mathrm{P}<0.05)$. There were 126 miRNAs with significant differences between MCF-7C and MCF-7T $(\mathrm{P}<0.05)$; among them, 76 miRNAs were upregulated $(\mathrm{P}<0.05)$ and 50 miRNAs were downregulated. On the basis of the results of the present study, we hypothesize that miR-21, miR-146a, miR-148a, miR-34a and miR-27a may serve important roles in mediating TAM resistance in breast cancer, and
\end{abstract}

Correspondence to: $\operatorname{Dr}$ Xinghua Long, Department of Laboratory Medicine, Zhongnan Hospital of Wuhan University, 169 Donghu Road, Wuchang, Wuhan, Hubei 430071, P.R. China E-mail: xlong888@yahoo.com

*Contributed equally

Key words: microRNA, breast cancer, MCF-7, tamoxifen, resistance have potential as therapeutic targets for TAM-resistant breast cancer.

\section{Introduction}

The treatment of breast cancer depends on its pathological type and estrogen receptor $\alpha(\mathrm{ER} \alpha)$ expression. Although the incidence of breast cancer has increased year by year among women in United States from 2004 to 2014, and has begun affecting younger age groups, the increased attention to (in terms of scientific studies) and subsequent improvements in the comprehensive treatment of breast cancer have resulted in decreases in breast cancer mortality in the United States over the past two decades (1). Among these improvements, endocrine therapy in breast cancer has received attention and has become the focus of numerous studies. A previous study demonstrated that $>70 \%$ of patients with breast cancer are $\mathrm{ER} \alpha$-positive $\left(\mathrm{ER} \alpha^{+}\right)$, affording clinicians the opportunity to provide adjuvant endocrine therapy (2). The selective estrogen receptor modulator tamoxifen (TAM) and aromatase inhibitors, including anastrozole, exemestane and letrozole, may inhibit disease recurrence in patients with $\mathrm{ER} \alpha^{+}$breast cancer, providing evidence of the involvement of $\operatorname{ER} \alpha$ in the pathogenesis of breast cancer $(3,4)$. However, a number of patients with breast cancer treated using endocrine therapy will develop resistance to these drugs, resulting in disease recurrence and leading to tumor progression. A previous study indicated that $\sim 40 \%$ of patients that received TAM or other endocrine treatment would experience disease recurrence (5). Prior studies have proposed a variety of possible mechanisms of TAM resistance in breast cancer, including ER and growth factor receptor interaction (6), and ER loss and mutation (7). However, the mechanism of endocrine resistance is complicated, and certain studies have identified that specific cases of secondary drug-resistant breast cancer remain $\mathrm{ER}^{+}(8,9)$. ER post-transcriptional modification is also considered to be one of the important mechanisms of TAM resistance (10). In addition, the abnormal expression of microRNAs (miRNAs/miRs) associated with drug resistance may lead to tumor cell drug resistance and secondary metastasis.

miRNAs are short, non-coding RNA molecules that regulate gene expression at the post-transcriptional level by directly binding to the 3'-untranslated region (3'-UTR) of the target mRNAs, causing translation inhibition, which is often 
accompanied by mRNA decay (11). Microarray technology is used to detect abnormal expression profiles of miRNAs in a range of types of cancer, such as breast cancer. A number of studies have demonstrated that miRNA expression profiles are associated with tumor grades and patient prognoses $(12,13)$. In cancer cells, miRNAs have been divided into oncogenic miRNAs and tumor suppressor miRNAs. Iorio et al (14) used microarrays containing all known miRNAs to quantify the expression levels of miRNAs in 76 breast tumors, and compared the results with those obtained from 34 normal breast tissue samples. The results indicated that the expression levels of 29 miRNAs changed significantly in breast cancer. Additional analysis indicated that miR-10b, miR-125b, miR-145, miR-21 and miR-155 were necessary to distinguish between cancerous and normal tissues, with miR-10b, miR-125b and miR-145 being significantly downregulated, and miR-21 and miR-155 being significantly upregulated (14). Iorio et al (14) demonstrated that these 5 miRNAs exhibited significant effects on the formation and growth of tumors, likely because they served a role in promoting or inhibiting the expression of estrogen and progesterone receptors in breast cancer. At present, the principle mechanism of TAM resistance in breast cancer remains unclear, and the mechanism of miRNA regulation has not been fully elucidated. Therefore, the present study initially established TAM-resistant breast cancer cell lines by two different methods, and identified novel avenues for reversing TAM resistance by using HiSeq 2500 sequencing technology to screen resistance-associated miRNAs.

The ER $\alpha$ expression level is an important indicator of prognosis and is associated with breast cancer drug resistance. It has been demonstrated that miRNA-206 expression is significantly reduced in $\mathrm{ER} \alpha^{+}$breast cancer (15); miR-206 may inhibit the expression of ER $\alpha$ on mRNA and protein levels, indicating that miRNA-206 could be used as a breast cancer endocrine therapy target (16). In addition, the study of TAM-resistant breast cancer cell lines has indicated that miR-221/miR-222 was significantly elevated in the TAM-resistant cell line (17). miR-221/miR-222 inhibited the expression of tumor suppressor cyclin-dependent kinase inhibitor 1B (p27 $\left.7^{\mathrm{kip} 1}\right)$, which resulted in $\mathrm{p} 27^{\mathrm{kip} 1}$ decline (17). With the reduction of $\mathrm{p} 27^{\mathrm{kip} 1}$, the migratory and invasive abilities of cells were increased, promoting the metastasis of breast cancer (18). The expression of miR-21 is associated with ER $\alpha$. A decrease in miR-21 expression led to the activation of expression of its target genes, programmed cell death 4 (PDCD4), phosphatase and tensin homolog and B-cell lymphoma 2 (Bcl-2), significantly increasing the sensitivity of tumor cells to aromatase inhibitors $(19,20)$. Concurrently, it was identified that the expression of miR-21 was positively correlated with the expression level of receptor tyrosine-protein kinase erbB-2 (HER2/neu). HER2/neu promotes the expression of miR-21 by activating the mitogen-activated protein kinase extracellular signal-related kinase 1/2 (MAPK ERK1/2) pathway, and the upregulation of miR-21 inhibits the expression of its target gene, PDCD4, a tumor metastasis suppressor protein (21). Therefore, the maintenance of expression and function of various proteins, including enzymes, depends on the specific regulation of miRNAs. If miRNA expression is abnormal, it will disrupt the homeostasis of gene expression, and lead to the abnormal expression of drug-associated genes, and finally result in the occurrence of drug resistance. In the present study, the human breast cancer MCF-7 cell line and two drug-resistant strains, MCF-7C and MCF-7T, were studied. miRNA expression was detected using the HiSeq 2500 sequencing system, identifying miRNAs with differential expression, which provide a basis for additional functional studies.

In the present study, two different drug-resistant breast cancer cell lines were established using two different methods of drug administration. Dose stepwise induction was used in the establishment of the MCF-7C line. Following each treatment with progressively increasing concentrations of 4-hydroxytamoxifen (OHT) screening medium, the drug-containing medium was replaced with drug-free complete medium, the apoptotic cells were removed, and the viable cells were allowed to proliferate. This process provided the appropriate time interval for tumor cells to activate relevant physiological pathways, including the Erk and PI3K pathways (10). Therefore, the tumor cells gradually adapted to the stimulation of drugs, and then obtain drug resistance. Drug-resistant cell lines established using gradual increases in drug concentration are considered to have acquired resistance during the processes of changing and remodeling of cell proliferation, cell cycle, apoptosis and genetics, consequently having acquired resistance in the process of screening and culturing (22). Therefore, we hypothesize that the MCF-7C line is a cell line with acquired drug-resistance. The other drug-resistant cell line, MCF-7T, was established by a temporal stepwise method, beginning with a high concentration of OHT. The culture time gradually increased from 1 to $48 \mathrm{~h}$, and then to $72 \mathrm{~h}$. The selection process provided a time interval that not only facilitated the repair of slightly damaged cells, but also favored the removal of cells that were relatively drug-sensitive, while cells that were inherently relatively resistant were retained. It is considered that a temporal stepwise method of inducing drug-resistant strains, beginning with high concentrations, preserved the cells with drug-resistant characteristics, namely the endogenous drug-resistant cells (23). Therefore, we hypothesized that the MCF-7T cell line was a combination of exogenous and endogenous resistance characteristics.

\section{Materials and methods}

Reagents. The human breast cancer MCF-7 cell line was purchased from the American Type Culture Collection (ATCC; Manassas, VA, USA). Dulbecco's modified Eagle's medium (DMEM), fetal bovine serum, trypsin and phosphate-buffered saline (PBS) were all purchased from Hyclone; GE Healthcare Life Sciences (Logan, UT, USA); OHT was purchased from Sigma-Aldrich; Merck KGaA (Darmstadt, Germany); EMD Millipore $^{\mathrm{TM}}$ Immobilon $^{\mathrm{TM}}$ Western Chemiluminescent HRP Substrate (ECL) was purchased from EMD Millipore (Billerica, MA, USA), and the rabbit anti-human ER (dilution 1:500; sc-543) and mouse anti-human GAPDH (dilution 1:500; sc-293335) antibodies were purchased from Santa Cruz Biotechnology, Inc. (Dallas, TX, USA).

Establishment of the TAM-resistant MCF-7C cell line using an OHT dose stepwise induction method. The MCF-7C cell line was established using OHT as the screening drug and the two-step screening method based on an OHT dose 
stepwise induction. This method was divided into two phases: Adaptation and consolidation. In the adaptation phase, MCF-7 cells were cultured to $70 \%$ confluence at $37^{\circ} \mathrm{C}$ in a humidified atmosphere containing $5 \% \mathrm{CO}_{2}$, then treated with $10 \mathrm{nM}$ OHT-containing DMEM for $72 \mathrm{~h}$ three times, with medium being removed and re-introduced between each step, followed by $20,50,100,200,500$ and $800 \mathrm{nM}$ OHT dose screens. In the consolidation phase, the cell line that was resistant to $800 \mathrm{nM}$ would be cultured in $1000 \mathrm{nM} \mathrm{OHT}$ medium at $37^{\circ} \mathrm{C}$ in a humidified atmosphere containing $5 \% \mathrm{CO}_{2}$ for $72 \mathrm{~h}$ and the medium was removed and re-introduced ten times. PBS was used for immediate washing following every change of medium. Following sequential treatment and culturing for a period of 1 year, until the cells were normalized in the selection culture medium ( $1 \mu \mathrm{M} \mathrm{OHT})$, the MCF-7C cell line was considered to be successfully established.

Establishment of the MCF-7T cell line (temporal stepwise induction with high concentration of OHT). The MCF-7T cell line was established by temporal stepwise induction, beginning with a high concentration of OHT, using the breast cancer MCF-7 cell line as the parental cell line and OHT as the screening agent. Again, the method was divided into two stages, adaptation and consolidation. MCF-7 cells were cultured for $1 \mathrm{~h}$ in $1 \mu \mathrm{M}$ OHT-containing DMEM three times, with medium being removed and re-introduced between each step, in total at $37^{\circ} \mathrm{C}$ in a humidified atmosphere containing $5 \% \mathrm{CO}_{2}$. Then, culture times of $2,4,12,24$ and $48 \mathrm{~h}$ were sequentially performed in $1 \mu \mathrm{M}$ OHT medium three times at $37^{\circ} \mathrm{C}$. In the consolidation stage, the cells obtained from the adaptation stage were cultured in $1 \mu \mathrm{M}$ OHT medium for $72 \mathrm{~h}$ and the $1 \mu \mathrm{M}$ medium was removed and re-introduced 10 times. PBS was used for immediate washing following every medium change. MCF-7T cell line was successfully established by continuous culture with $1 \mu \mathrm{M}$ OHT.

Expression of ER protein was detected by western blot analysis. Proteins from MCF-7 cells and the drug-resistant sub-clones were harvested using immunoprecipitation assay lysis buffer (Beyotime Institute of Biotechnology, Haimen, China) and protein samples were prepared through sonication at $20 \mathrm{KHz}$ and the concentration was determined by BCA method. Protein samples $(50 \mu \mathrm{g})$ were subjected to $8 \%$ SDS-PAGE, and the proteins were then transferred from the gel to a polyvinylidene fluoride membrane, the membrane was next blocked in TBS with Tween-20 with 5\% skim milk (BD Biosciences, Franklin Lakes, NJ, USA) at room temperature for $2 \mathrm{~h}$, followed by incubation with the ER antibody (dilution 1:500) at $4^{\circ} \mathrm{C}$ overnight then with the mouse anti-rabbit secondary antibody sc-2357 (dilution 1:500; Santa Cruz Biotechnology, Inc.) at room temperature for $2 \mathrm{~h}$. Bands were detecting using the ECL reagent, developed with X-ray film and scanned using Gel Doc ${ }^{\mathrm{TM}} \mathrm{XR}+$ Imaging System (Bio-Rad Laboratories, Inc., Hercules, CA, USA) to obtain image data. Finally, the expression level of the target protein was obtained through AlphaEaseFC version 6.0 (ProteinSimple, San Jose, CA, USA) image analysis.

Total cellular RNA extraction. Cells in the log growth phase were harvested and TRIzol ${ }^{\circledR}$ (Life Technologies; Thermo
Fisher Scientific, Inc., Waltham, MA, USA) was used to isolate RNA at $4{ }^{\circ} \mathrm{C}$ according to the manufacturer's instructions. The separated RNA was dissolved in diethyl pyrocarbonate water and diluted to $500 \mathrm{ng} / \mu \mathrm{l}$. The concentration and purity of RNA was quantified using a NanoDrop 2000 Ultraviolet Spectrophotometer (NanoDrop Technologies; Thermo Fisher Scientific, Inc., Pittsburgh, PA, USA). Samples with an optical density (OD)260/OD280 ratio of 1.8-2.0 were considered to have met the requirements.

Library construction and sequencing. Small RNA high-throughput sequencing was performed by Beijing Biomarker Biotechnology Co., Ltd. (Beijing, China), and the library was constructed and sequenced as follows: $1.5 \mu \mathrm{g}$ RNA was used as the initial amount of the RNA sample and the volume of the sample was supplemented with water to make a total reaction volume of $6 \mu 1$. Next, the library was constructed using the small RNA Sample Prep kit (New England Biolabs, Ltd., Beijing, China), according to the manufacturer's instructions. Subsequent to the construction of the library, the concentration of the samples was determined by Qubit 2.0 (Life Technologies; Thermo Fisher Scientific, Inc.) and the samples within the library were diluted to a concentration of $1 \mathrm{ng} / \mu \mathrm{l}$. The insert size was then examined using the Agilent 2100 Bioanalyzer (Agilent Technologies, Inc., Santa Clara, CA, USA). High-throughput sequencing of the library was performed using the HiSeq 2500 library with sequencing read length single-end (SE) of $50 \mathrm{nt}$ at Biomarker Biotechnology Co., Ltd.

miRNA analysis and identification. As miRNA transcription initiation sites are primarily located in the gene spacer, intron and reverse complement sections of the coding sequence, the precursors have a marked hairpin structure that is formed by the cleavage of the endoribonuclease Dicer/Dicer-like enzyme (24). On the basis of the distribution of length of reads on the precursor sequence (based on the characteristics of the miRNA, mature, star, loop sections) and the energy information of the precursor structure, which is based on the sequence of reads (RNA-fold), miRDeep2 software version 2.0.0.8 (Max Delbrück Center for Molecular Medicine, Berlin, Germany) was used to identify known and novel miRNAs.

Differential expression profiling of miRNA. miRDeep2 software was used to identify known miRNAs and predict novel miRNAs by alignment to the reference genome GRCh37 (http://grch37.ensembl.org/Homo_sapiens/Info/Index). The expression of miRNAs in each sample was analyzed statistically using EBseq R package version 1.19.0 (Bioconductor; https://www.bioconductor.org/help/) and the expression was normalized using a Transcript Per Million algorithm (25). In the process of differential expression miRNA detection, $\log _{2}$ fold change $(\mathrm{FC}) \mid \geq 1$ and false discovery rate $(\mathrm{FDR}) \leq 0.01$ were used as the screening criteria. The FC represents the ratio of expression between two samples (groups). The P-value of the original hypothesis may be expressed as the probability of non-discrepancy. As the differential expression analysis of miRNAs is an independent statistical hypothesis test for a large number of miRNA expression, there will be a false-positive issue. Therefore, in the process of analysis, the 


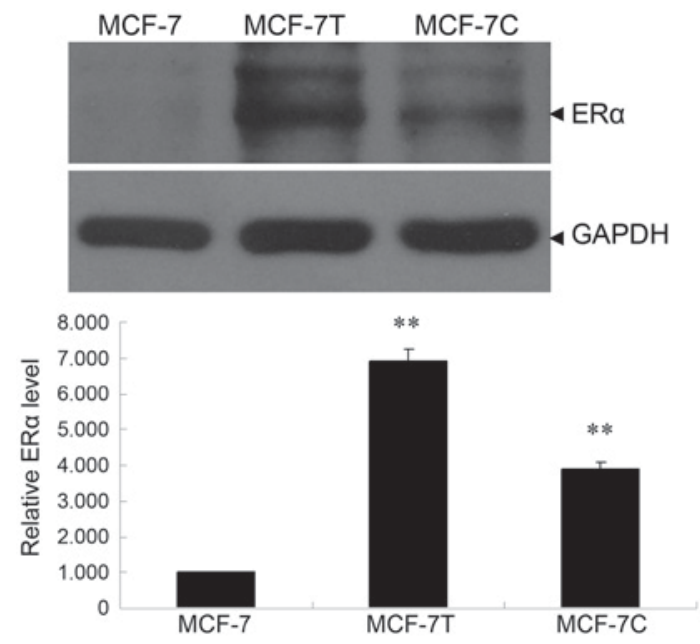

Figure 1. ER $\alpha$ expression in MCF-7, MCF-7T and MCF-7C cell lines. (A) Western blot analysis and (B) quantitative analysis of western blot analysis normalized to GAPDH. Data presented represent the results of two independent experiments, each performed in duplicate. ${ }^{* *} \mathrm{P}<0.01$ compared with MCF-7. ER $\alpha$, estrogen receptor- $\alpha$.

Benjamini-Hochberg correction method was used to calculate the P-value, and the FDR was used as the key indicator of differential expression profiling of miRNA to identify the differentially expressed genes (DEGs). Hierarchical cluster analysis of DEGs was performed using TMeV version 4.9.0 (GitHub, Inc., San Francisco, CA, USA).

Statistical analysis. Data are presented as the mean \pm standard deviation. Statistical differences between groups in western blot analysis were assessed using analysis of variance with a post hoc least significant difference test on SPSS version 22 (IBM Corp., Armonk, NY, USA). P $<0.05$ was considered to indicate a statistically significant difference.

\section{Results}

Western blotting detects ER $\alpha$ protein in MCF-7, MCF-7C and $M C F-7 T$ cells. The protein expression of ER $\alpha$ was detected by western blotting. The expression of ER $\alpha$ in MCF-7C and MCF-7T cells was significantly increased, compared with MCF-7 cells (Fig. 1; $\mathrm{P}<0.01$ ).

MiRNA differential expression analysis. In the present study, the expression levels of miRNAs in MCF-7 cells and TAM-resistant breast cancer cell lines were assessed using HiSeq 2500 sequencing technology. The results indicated that 1,646 miRNAs were detected in the 3 cell samples, including 1,376 known miRNAs and 270 predicted miRNAs. In the process of differential expression miRNA detection, $\log _{2}$ $\mathrm{FCl} \geq 1$ and $\mathrm{FDR} \leq 0.01$ was used as the screening criteria. The results indicated that there were 118 miRNAs that exhibited significant differences in expression between MCF-7C and MCF-7 cells $(\mathrm{P}<0.05)$; among them, there were 67 upregulated and 51 downregulated miRNAs. Upregulated miRNAs in MCF-7C included let-7c, let-7e, miR-21 and miR-486, whereas downregulated miRNAs included miR-146a, miR-148a and miR-342. There were 42 miRNAs that exhibited significant differences in expression between MCF-7T
Table I. Differentially expressed miRNA.

\begin{tabular}{lrcc}
\hline DEG group & $\begin{array}{r}\text { Total } \\
\text { DEG }\end{array}$ & Upregulated & Downregulated \\
\hline MCF-7C vs. MCF-7 & 118 & 67 & 51 \\
MCF-7T vs. MCF-7 & 42 & 23 & 19 \\
MCF-7C vs. MCF-7T & 126 & 76 & 50 \\
\hline
\end{tabular}

DEG, differentially expressed genes; miRNA, microRNA.

and MCF-7 $(\mathrm{P}<0.05)$; among them there were 23 upregulated and 19 downregulated miRNAs. Upregulated miRNAs in MCF-7T included let-7i, miR-145, miR-199b and miR-486, whereas downregulated miRNAs included miR-146a and miR-27a. Compared with the drug-resistant MCF-7T cell line, 126 miRNAs were expressed at significantly different levels in MCF-7C cells $(\mathrm{P}<0.05)$; among them there were 76 upregulated and 50 downregulated miRNAs. Upregulated miRNAs in MCF-7C included let-7c-3p and let-7e-5p, whereas the downregulated miRNAs included miR-145-5p, miR-148a-5p and miR-199b. The number of DEGs is summarized in Table I.

The differentially expressed miRNAs exhibiting the same or similar expression behavior were clustered by hierarchical cluster analysis. The cluster results of differentially expressed miRNAs are presented in Fig. 2. Certain differentially expressed miRNAs in the two drug-resistant breast cancer cell lines are summarized in Table II.

\section{Discussion}

miRNAs have become the focus of current oncology studies. A previous study have identified that miRNA may regulate gene expression, and affect the development, metabolism and other physiological processes of disease (11). microRNAs are short non-coding RNA molecules that regulate gene expression at post-transcriptional levels by directly targeting the 3'-UTR of target mRNAs. These molecules participate in the regulation of the signaling pathways and genes involved in tumorigenesis and progression, thereby affecting cellular processes such as differentiation, migration and apoptosis (26). A large number of studies have indicated that miRNAs may regulate the expression of oncogenes or tumor suppressor genes, and serve the roles of oncogenes or tumor suppressor genes (27). miRNAs are aberrantly expressed in a variety of cancer cells, including those of breast cancer (28). Previously, studies have indicated that miRNAs are not only closely associated with the occurrence and development of tumors, but also serve a notable role in the development of drug resistance (29). It is speculated that the differential expression of miRNA may also be involved in the regulation of TAM resistance in breast cancer. At present, little is known about miRNAs in TAM-resistant breast cancer. miRNA detection methods include miRNA microarrays and small RNA sequencing. Compared with microRNA microarrays, microRNA sequencing has a number of advantages, including increased data throughput capacity, higher sensitivity and accuracy, wider dynamic detection range, more 


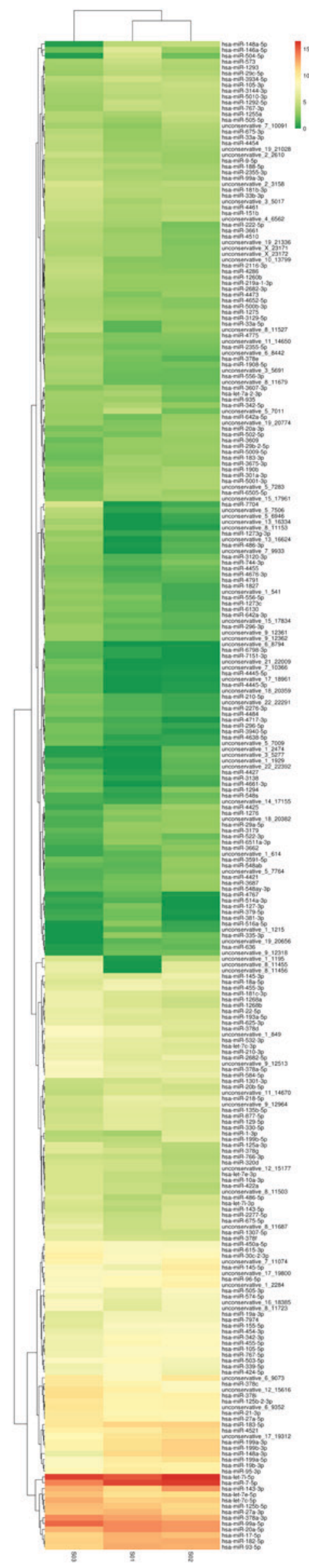

Figure 2. Differentially expressed miRNA clusters in MCF-7 (S01), MCF-7T (S02) and MCF-7C (S03) cell lines. Hsa, Homo sapiens; miR, microRNA. abundant content of bioinformatics analysis and the ability to detect unknown microRNAs (30).

The let-7 miRNA family was the second miRNA to be identified (31), and is one of the most widely-studied miRNAs. The let-7 family is highly conserved in terms of sequence and functions, and its expression in tumor cells is usually reduced (32). Let-7 may regulate the epithelial-mesenchymal transition (EMT) of tumor cells (33). Low let-7 expression is usually characterized by mesenchymal cells. High expression of let-7 is a characteristic of epithelial cells. The expression of let-7 is closely associated with the malignancy of tumors. As a tumor suppressor gene, it serves an important role in cell growth, differentiation and apoptosis, primarily through the regulation of proto-oncogenes or transcription factors (34). For example, a decrease in let-7 expression in breast cancer stem cells results in an overexpression of the RAS proto-oncogenes (35). Estrogen receptor signaling may induce the expression of let-7a, while downregulating the expression of its target genes, v-myc avian myelocytomatosis viral oncogene homolog MYC and K-Ras, inhibits tumor cell proliferation (36). Chen et al (20) identified that the expression of ER $\alpha-36$ (a 36-kDa novel isoform of ER-a66) was downregulated by let-7 in mammary glands, which decreased the phosphorylation of ERK and RAC serine/threonine-protein kinase (Akt), and affected the MAPK/ERK and phosphoinositide 3-kinase (PI3K)/Akt signaling pathways. In TAM-resistant breast cancer cells, increasing the expression of let-7 may reduce the expression of ER- $\alpha 36$, thereby enhancing the sensitivity of tumor cells to drugs (37). However, the high expression of let-7 in the MCF-7C and MCF-7T TAM-resistant cell lines is not consistent with the previous study (37). The high expression of let-7 may be explained by high ER $\alpha$ protein expression; however, this mechanism in tumor cell resistance requires additional study and verification.

It has been demonstrated that miR-21 serves as a proto-oncogene and inhibits the expression of the pro-apoptotic gene Bcl-2, thus affecting tumor growth (19). In addition, miR-21 may regulate the metastasis and infiltration of cells by regulating a variety of tumor metastasis suppressor genes such as PDCD4 and tropomyosin $1 \alpha$ (38). The role of miR-21 in tumor drug resistance has attracted attention. Blower et al (39) demonstrated that the expression of miR-21 was associated with chemotherapeutic efficacy, and that reducing miR-21 levels improved the efficacy of multiple drugs, indicating that miR-21 expression is associated with drug resistance in breast cancer cells. miR-21 is highly expressed in adriamycin-resistant breast cancer cells, and the upregulation or downregulation of miR-21 may affect the sensitivity of breast cancer cells to doxorubicin (40). The results of the present study indicated that miR-21 expression in TAM-resistant cells was significantly increased. This is consistent with the findings of previous study, which demonstrated that estradiol inhibits miR-21 expression and estrogen antagonists may increase miR-21 expression, indicating that miR-21 and ER may interact with each other (19). Therefore, decreasing the expression of miR-21 may be a novel method of improving the treatment of patients with breast cancer. Lee et al (41) and Ota et al (42) suggested that there was a significant positive association between miR-21 expression and HER-2 status. As a commonly used marker for breast cancer, HER-2 is believed 
Table II. Expression of certain miRNAs in two drug-resistant breast cancer cell lines.

\begin{tabular}{|c|c|c|c|c|c|c|}
\hline \multirow[b]{2}{*}{ DEG } & \multicolumn{3}{|c|}{ MCF-7C vs. MCF-7 } & \multicolumn{3}{|c|}{ MCF-7T vs. MCF-7 } \\
\hline & $\log 2 \mathrm{FC}$ & P-value & Regulated & $\log 2 \mathrm{FC}$ & P-value & Regulated \\
\hline Let-7c & 1.1522 & 0 & Up & - & - & - \\
\hline Let-7e & 1.3271 & 0 & Up & - & - & - \\
\hline Let-7i & 1.0847 & 0 & Up & 1.2287 & 0 & Up \\
\hline $\operatorname{miR}-145-5 p$ & - & - & - & 2.7938 & 0 & Up \\
\hline miR-486-5p & 1.9927 & 0 & Up & 1.3708 & 0 & Up \\
\hline miR-21-3p & 1.1983 & 0 & Up & - & - & - \\
\hline miR-146a-5p & -4.2962 & 0 & Down & -3.0789 & 0 & Down \\
\hline $\operatorname{miR}-148 a-5 p$ & -6.1588 & 0 & Down & - & - & - \\
\hline miR-27a & - & - & - & -1.0310 & 0 & Down \\
\hline miR-199b & -1.4658 & 0 & Down & 2.1652 & 0 & Up \\
\hline
\end{tabular}

DEG, differentially expressed genes; miRNA/miR, microRNA; FC, fold change.

to promote tumor metastasis. Huang et al (21) demonstrated that HER-2 may increase the expression of miR-21 through the MAPK signaling pathway, promoting cell invasion. As ER and HER-2 are important indicators of endocrine therapy for breast cancer, indicating that miR-21 could be used as a predictor of endocrine therapy.

Previous studies demonstrated that miR-486 is downregulated in the majority of tumors (43-45). Zhang et al (46) identified that miR-486 was significantly downregulated in breast cancer tissues and cell lines. The overexpression of miR-486 may significantly inhibit the proliferation of breast cancer cells, induce $\mathrm{G}_{0} / \mathrm{G}_{1}$ arrest and promote cell apoptosis. Additionally, it was confirmed that the overexpression of proto-oncogene serine-threonine-protein kinase (PIM-1) attenuates the inhibitory effect of miR-486 on breast cancer cells (43). It was deduced that miR-486 may serve an anti-proliferative role by directly downregulating the expression of PIM-1 (46). Therefore, the interaction between the miR-486/PIM-1 axis may contribute to the understanding of the mechanism of drug resistance in breast cancer.

It has been identified that the overexpression of miR-146a in the highly metastatic human breast cancer MDA-MB-231 cell line significantly downregulated interleukin-1 receptor-associated kinase 1 and tumor necrosis factor receptor associated factor 6 expression, and negatively regulated nuclear factor $-\kappa \mathrm{B}(\mathrm{NF}-\kappa \mathrm{B})$ activity, significantly reducing the invasive and migratory capacity of the cells (47). These data indicate that miR-146a is a negative regulator of $N F-\kappa B$ activity in human breast cancer cells, and the low miR-146a expression exhibited in the two drug-resistant cell lines in the present study indicating that the modulation of miR-146a levels may have potential therapeutic significance by inhibiting breast cancer metastasis and drug resistance.

Insulin receptor substrate-1 (IRS-1) and insulin-like growth factor I receptor (IGF-IR) induce multiple signaling cascades such as PI3K/AKT and MAPK/ERK, and control tumor formation and progression (48). miR-148a may inhibit the proliferation of breast cancer cells by regulating IRS-1 and IGF-IR. The upregulation of miR-148a may specifically inhibit the expression of IGF-IR/PKM2. miR-148a may inhibit tumor metastasis and invasion by the regulation of specific target genes, including hematopoietic-related PBX interacting proteins, matrix metalloproteinase 7 , ubiquitin-specific protease 4, HOX transcriptional antisense RNA and S-phase kinase-associated protein 1. miR-148a may inhibit Bcl-2-induced tumor cell apoptosis (49). In the present study, when the expression of miR-148a in TAM-resistant cell lines was downregulated, the tumor inhibition in metastasis and invasion was weakened and the level of drug resistance was increased.

The results of sequencing in the present study indicated that miR-342 expression was significantly decreased in the TAM-resistant cell lines, which is consistent with the results of a previous study (50). Cittelly et al (50) identified that the inhibition of miR-342 expression incurred resistance to TAM in the breast cancer MCF-7 cell line. Conversely, by increasing the expression of miR-342, the rate of resistance development to endocrine therapy may be decreased and rates of apoptosis are increased in tumor cells (50); therefore, the tumor cells become sensitive to the drug treatment. miR-342 is aberrantly expressed in a variety of tumor cell models and the expression of miR-342 is associated with the expression of its host gene, Ena/vasodilator-stimulated phosphoprotein-like (EVL). It was identified that miR-342 was highly expressed in $\mathrm{ER} \alpha^{+}$ breast cancer cells and decreased in ER $\alpha$ negative cells (51). However, EVL expression was significantly lower in ER $\alpha^{+}$ breast cancer cells that failed to respond to TAM treatment, compared to TAM-sensitive ER $\alpha^{+}$breast cancer cells (50), so it was hypothesized that TAM-induced tumor cell resistance may be induced by inhibiting miR-342 expression. miR-342 may serve an important role in promoting cell apoptosis. miR-342 may be involved in the regulation of the mitotic-like POLO-like kinase signaling pathway and cell cycle-associated signaling pathway-associated proteins, such as cyclin B1 and tumor protein P53 (50). It has also been demonstrated that miR-342 may affect chemosensitivity by modulating histone methylation (52). Therefore, miR-342 may affect the sensitivity of MCF-7 cells to TAM, indicating that miR-342 may 
serve as a molecular marker for predicting TAM sensitivity in $\mathrm{ER}^{+}$breast cancer, and may be a potential target for endocrine adjuvant therapy.

In addition, the results of the sequencing in the present study indicated that miR-27a was downregulated in the TAM-resistant cell lines. Feng et al (53) identified that miR-27a could bind to the 3'-UTR of the adenosine 5-triphosphate binding cassette subfamily $B$ member 1 gene and inhibit its expression, and therefore may directly regulate the expression of the multidrug resistance protein P-glycoprotein. Concurrently, the overexpression of miR-27a in leukemia cells may increase chemosensitivity in leukemia cells. We hypothesized that inducible resistance as a result of low miR-27a expression in breast cancer cells may be associated with this mechanism.

Expression of miR-145 was upregulated in the TAM-resistant cell lines in the present study. Nam et al (54) suggested that miR-145 may target the upstream regulatory factors IGF-1R and signal transducer and activator of transcription 1 (STAT1) in glycolysis, thereby affecting cell metabolism and the expression of the Janus kinase/STAT signaling pathway, consequently regulating EMT and tumor metastasis.

The drug-resistant MCF-7C and MCF-7T cell lines exhibited different miRNA expression profiles, indicating that these drug-resistant cell lines have different molecular mechanisms of drug resistance. As they were established using different modes of drug administration, we hypothesize that TAM administration in the treatment of patients with breast cancer has an effect on the formation of drug resistance. Silencing of miR-199b-5p due to epigenetic changes, including promoter CpG island methylation, activates the JAG1-mediated Notch1 signaling cascade, leading to the development of acquired resistance in ovarian cancer (55). The expression of miR-199b-5p was downregulated in the MCF-7C cell line and upregulated in the MCF-7T cell line, indicating that MCF-7C may be a cell line that acquired drug-resistance by a dose stepwise induction method, which was consistent with a previous study (55). These results indicate that miR-199b-5p may be a novel therapeutic target for breast cancer therapy.

In the present study, drug resistance in MCF-7C cells was established by dose stepwise induction and in MCF-7T cells by a temporal stepwise method. The mechanism of drug resistance exhibited by $\mathrm{MCF}-7 \mathrm{C}$ cells may differ from that of MCF-7T cells. MCF-7C may be an acquired drug resistant cell line, and MCF-7T may be a combination of acquired resistance cells and endogenous resistant cells. A clearer and more detailed drug resistance mechanism requires further characterization. However, at present the two cell lines may only be distinguished by clinical concepts; there is a lack of specific criteria to determine whether in vitro-established drug-resistant cell lines exhibit endogenous or acquired resistance. The establishment of drug-resistant cancer cell lines is designed to provide the correct resources for overcoming clinical drug resistance to breast cancer. Therefore, the specific association between endogenous and acquired resistance requires additional study. The establishment of appropriate drug-resistant cancer cell lines will provide useful information for elucidating the mechanisms of tumor drug resistance, and thus methods for clinical treatment.
The expression of ER in MCF-7C and MCF-7T cells was significantly increased compared with that in the MCF-7 cells, indicating that the acquisition of TAM resistance in tumor cells may be independent of ER loss. On the basis of the results of the present and previous studies, we hypothesize that miR-21, miR-146a, miR-148a, miR-34a and miR-27a serve important roles in TAM resistance in breast cancer, and may be potential targets for breast cancer treatment. The roles of miR-486, miR-505, miR-29 and miR-145 require additional study. In the present study, the differentially expressed miRNAs associated with drug resistance in breast cancer cells were obtained by RNA sequencing technology, providing data for future studies and a basis for the identification of the mechanism of drug resistance and reversal of tumor drug resistance.

\section{Acknowledgements}

The present study was supported by grants from National Natural Science Foundation of China (grant nos. 81272372 and 30873044). The Project-sponsored by Scientific Research Foundation for Returned Overseas Chinese Scholars, State Education Ministry.

\section{References}

1. Siegel RL, Miller KD and Jemal A: Cancer Statistics, 2017. CA Cancer J Clin 67: 7-30, 2017.

2. Rao X, Di Leva G, Li M, Fang F, Devlin C, Hartman-Frey C, Burow ME, Ivan $M$, Croce $C M$ and Nephew KP: MicroRNA-221/222 confers breast cancer fulvestrant resistance by regulating multiple signaling pathways. Oncogene 30 : 1082-1097, 2011.

3. Wolmark N, Mamounas EP, Baehner FL, Butler SM, Tang G, Jamshidian F, Sing AP, Shak S and Paik S: Prognostic impact of the combination of recurrence score and quantitative estrogen receptor expression (ESR1) on predicting late distant recurrence risk in estrogen receptor-positive breast cancer after 5 years of Tamoxifen: Results from NRG oncology/national surgical adjuvant breast and bowel project B-28 and B-14. J Clin Oncol 34: 2350-2358, 2016.

4. Ingle JN: Postmenopausal women with hormone receptor-positive breast cancer: Balancing benefit and toxicity from aromatase inhibitors. Breast 22 (Suppl 2): S180-S183, 2013.

5. Manavalan TT, Teng Y, Appana SN, Datta S, Kalbfleisch TS, Li Y and Klinge CM: Differential expression of microRNA expression in tamoxifen-sensitive MCF-7 versus tamoxifen-resistant LY2 human breast cancer cells. Cancer Lett 313: 26-43, 2011.

6. Arpino G, Wiechmann L, Osborne CK and Schiff R: Crosstalk between the estrogen receptor and the HER tyrosine kinase receptor family: Molecular mechanism and clinical implications for endocrine therapy resistance. Endocr Rev 29: 217-233, 2008.

7. Friedman RC, Farh KK, Burge CB and Bartel DP: Most mammalian mRNAs are conserved targets of microRNAs. Genome Res 19: 92-105, 2009.

8. Ciupek A, Rechoum Y, Gu G, Gelsomino L, Beyer AR, Brusco L, Covington KR, Tsimelzon A and Fuqua SA: Androgen receptor promotes tamoxifen agonist activity by activation of EGFR in ERalpha-positive breast cancer. Breast Cancer Res Treat 154: 225-237, 2015.

9. Karam M, Bièche I, Legay C, Vacher S, Auclair C and Ricort JM: Protein kinase D1 regulates ER $\alpha$-positive breast cancer cell growth response to $17 \beta$-estradiol and contributes to poor prognosis in patients. J Cell Mol Med 18: 2536-2552, 2014.

10. Musgrove EA and Sutherland RL: Biological determinants of endocrine resistance in breast cancer. Nat Rev Cancer 9: 631-643, 2009.

11. Ambros V: The functions of animal microRNAs. Nature 431: 350-355, 2004.

12. Ma L, Teruya-Feldstein J and Weinberg RA: Tumour invasion and metastasis initiated by microRNA-10b in breast cancer. Nature 449: 682-688, 2007. 
13. Cancer Genome Atlas Network: Comprehensive molecular portraits of human breast tumours. Nature 490: 61-70, 2012.

14. Iorio MV,Ferracin M, Liu CG, Veronese A, Spizzo R, Sabbioni S, Magri E, Pedriali M, Fabbri M, Campiglio M, et al: MicroRNA gene expression deregulation in human breast cancer. Cancer Res 65: 7065-7070, 2005

15. Kondo N, Toyama T, Sugiura H, Fujii Y and Yamashita H: miR-206 expression is down-regulated in estrogen receptor alpha-positive human breast cancer. Cancer Res 68: 5004-5008, 2008.

16. Adams BD, Furneaux H and White BA: The micro-ribonucleic acid (miRNA) miR-206 targets the human estrogen receptor-alpha (ERalpha) and represses ERalpha messenger RNA and protein expression in breast cancer cell lines. Mol Endocrinol 21: $1132-1147,2007$

17. Miller TE, Ghoshal K, Ramaswamy B, Roy S, Datta J, Shapiro CL, Jacob S and Majumder S: MicroRNA-221/222 confers tamoxifen resistance in breast cancer by targeting $\mathrm{p} 27^{\mathrm{Kipl}}$. J Biol Chem 283: 29897-29903, 2008.

18. Yuan Y, Qin L, Liu D, Wu RC, Mussi P, Zhou S, Songyang Z and $\mathrm{Xu} \mathrm{J}$ : Genetic screening reveals an essential role of $\mathrm{p} 27^{\mathrm{kip} 1}$ in restriction of breast cancer progression. Cancer Res 67: 8032-8042, 2007.

19. Wickramasinghe NS, Manavalan TT, Dougherty SM, Riggs KA, Li Y and Klinge CM: Estradiol downregulates miR-21 expression and increases miR-21 target gene expression in MCF-7 breast cancer cells. Nucleic Acids Res 37: 2584-2595, 2009.

20. Chen Z, Yuan YC, Wang Y, Liu Z, Chan HJ and Chen S: Down-regulation of programmed cell death 4 (PDCD4) is associated with aromatase inhibitor resistance and a poor prognosis in estrogen receptor-positive breast cancer. Breast Cancer Res Treat 152: 29-39, 2015.

21. Huang TH, Wu F, Loeb GB, Hsu R, Heidersbach A, Brincat A, Horiuchi D, Lebbink RJ, Mo YY, Goga A and McManus MT: Up-regulation of miR-21 by HER2/neu signaling promotes cell invasion. J Biol Chem 284: 18515-18524, 2009.

22. Valeri N, Gasparini P, Braconi C, Paone A, Lovat F, Fabbri M, Sumani KM, Alder H, Amadori D, Patel T, et al: MicroRNA-21 induces resistance to 5-fluorouracil by down-regulating human DNA MutS homolog 2 (hMSH2). Proc Natl Acad Sci USA 107: 21098-21103, 2010.

23. Yang LY and Trujillo JM: Biological characterization of multidrug-resistant human colon carcinoma sublines induced/selected by two methods. Cancer Res 50: 3218-3225, 1990.

24. Ha M and Kim VN: Regulation of microRNA biogenesis. Nat Rev Mol Cell Biol 15: 509-524, 2014.

25. Fahlgren N, Howell MD, Kasschau KD, Chapman EJ, Sullivan CM, Cumbie JS, Givan SA, Law TF, Grant SR, Dangl JL and Carrington JC: High-throughput sequencing of Arabidopsis microRNAs: Evidence for frequent birth and death of MIRNA genes. PLoS One 2: e219, 2007.

26. Hammond SM: RNAi, microRNAs, and human disease. Cancer Chemother Pharmacol 58 (Suppl 1): S63-S68, 2006.

27. Bajan S and Hutvagner G: Regulation of miRNA processing and miRNA mediated gene repression in cancer. Microrna 3: 10-17, 2014.

28. Nygaard S, Jacobsen A, Lindow M, Eriksen J, Balslev E, Flyger H, Tolstrup N, Møller S, Krogh A and Litman T: Identification and analysis of miRNAs in human breast cancer and teratoma samples using deep sequencing. BMC Med Genomics 2: 35, 2009.

29. Yahya SM and Elsayed GH: A summary for molecular regulations of miRNAs in breast cancer. Clin Biochem 48: 388-396, 2015

30. Rai G, Rai R, Saeidian AH and Rai M: Microarray to deep sequencing: Transcriptome and miRNA profiling to elucidate molecular pathways in systemic lupus erythematosus. Immunol Res 64: 14-24, 2016

31. Reinhart BJ, Slack FJ, Basson M, Pasquinelli AE, Bettinger JC, Rougvie AE, Horvitz HR and Ruvkun G: The 21-nucleotide let-7 RNA regulates developmental timing in Caenorhabditis elegans. Nature 403: 901-906, 2000

32. Dahiya N, Sherman-Baust CA, Wang TL, Davidson B, Shih IeM, Zhang Y, Wood W III, Becker KG and Morin PJ: MicroRNA expression and identification of putative miRNA targets in ovarian cancer. PLoS One 3: e2436, 2008.

33. Jin B, Wang W, Meng XX, Du G, Li J, Zhang SZ, Zhou BH and Fu ZH: Let-7 inhibits self-renewal of hepatocellular cancer stem-like cells through regulating the epithelial-mesenchymal transition and the Wnt signaling pathway. BMC Cancer 16: 863, 2016.

34. Shell S, Park SM, Radjabi AR, Schickel R, Kistner EO, Jewell DA, Feig C, Lengyel E and Peter ME: Let-7 expression defines two differentiation stages of cancer. Proc Natl Acad Sci USA 104: 11400-11405, 2007.
35. Sun X, Fan C, Hu LJ, Du N, Xu CW and Ren H: Role of let-7 in maintaining characteristics of breast cancer stem cells. Xi Bao Yu Fen Zi Mian Yi Xue Za Zhi 28: 789-792, 2012 (In Chinese).

36. Barekati Z, Radpour R, Lu Q, Bitzer J, Zheng H, Toniolo P, Lenner $\mathrm{P}$ and Zhong XY: Methylation signature of lymph node metastases in breast cancer patients. BMC Cancer 12: 244 2012.

37. Zhao Y, Deng C, Lu W, Xiao J, Ma D, Guo M, Recker RR, Gatalica Z, Wang Z and Xiao GG: let-7 microRNAs induce tamoxifen sensitivity by downregulation of estrogen receptor $\alpha$ signaling in breast cancer. Mol Med 17: 1233-1241, 2011.

38. Qi L, Bart J, Tan LP, Platteel I, Sluis Tv, Huitema S, Harms G, $\mathrm{Fu} \mathrm{L}$, Hollema $\mathrm{H}$ and Berg Av: Expression of miR-21 and its targets (PTEN, PDCD4, TM1) in flat epithelial atypia of the breast in relation to ductal carcinoma in situ and invasive carcinoma. BMC Cancer 9: 163, 2009.

39. Blower PE, Chung JH, Verducci JS, Lin S, Park JK, Dai Z, Liu CG, Schmittgen TD, Reinhold WC, Croce CM, et al: MicroRNAs modulate the chemosensitivity of tumor cells. Mol Cancer Ther 7: 1-9, 2008.

40. Wang ZX,Lu BB, Wang H, Cheng ZX and Yin YM: MicroRNA-21 modulates chemosensitivity of breast cancer cells to doxorubicin by targeting PTEN. Arch Med Res 42: 281-290, 2011.

41. Lee JA, Lee HY, Lee ES, Kim I and Bae JW: Prognostic implications of microRNA-21 overexpression in invasive ductal carcinomas of the breast. J Breast Cancer 14: 269-275, 2011.

42. Ota D, Mimori K, Yokobori T, Iwatsuki M, Kataoka A, Masuda N, Ishii $\mathrm{H}$, Ohno $\mathrm{S}$ and Mori M: Identification of recurrence-related microRNAs in the bone marrow of breast cancer patients. Int J Oncol 38: 955-962, 2011.

43. Tian F, Shen Y, Chen Z, Li R, Lu J and Ge Q: Aberrant miR-181b-5p and miR-486-5p expression in serum and tissue of non-small cell lung cancer. Gene 591: 338-343, 2016.

44. Shindo Y, Hazama S, Nakamura Y, Inoue Y, Kanekiyo S, Suzuki N, Takenouchi H, Tsunedomi R, Nakajima M, Ueno T, et al: miR-196b, miR-378a and miR-486 are predictive biomarkers for the efficacy of vaccine treatment in colorectal cancer. Oncol Lett 14: 1355-1362, 2017.

45. Ren C, Chen H, Han C, Fu D, Zhou L, Jin G, Wang F, Wang D, Chen Y, Ma L, et al: miR-486-5p expression pattern in esophageal squamous cell carcinoma, gastric cancer and its prognostic value. Oncotarget 7: 15840-15853, 2016.

46. Zhang G, Liu Z, Cui G, Wang X and Yang Z: MicroRNA-486-5p targeting PIM-1 suppresses cell proliferation in breast cancer cells. Tumour Biol 35: 11137-11145, 2014.

47. Rusca N and Monticelli S: MiR-146a in immunity and disease. Mol Biol Int 2011: 437301, 2011.

48. Madhala-Levy D, Williams VC, Hughes SM, Reshef R and Halevy O: Cooperation between Shh and IGF-I in promoting myogenic proliferation and differentiation via the MAPK/ERK and PI3K/Akt pathways requires Smo activity. J Cell Physiol 227: 1455-1464, 2012

49. Li Y, Deng X, Zeng X and Peng X: The role of mir-148a in cancer. J Cancer 7: 1233-1241, 2016.

50. Cittelly DM, Das PM, Spoelstra NS, Edgerton SM, Richer JK, Thor AD and Jones FE: Downregulation of miR-342 is associated with tamoxifen resistant breast tumors. Mol Cancer 9: 317, 2010.

51. Farmer P, Bonnefoi H, Becette V, Tubiana-Hulin M, Fumoleau P, Larsimont D, Macgrogan G, Bergh J, Cameron D, Goldstein D, et al: Identification of molecular apocrine breast tumours by microarray analysis. Oncogene 24 : 4660-4671, 2005.

52. Jordan MA and Wilson L: Microtubules as a target for anticancer drugs. Nat Rev Cancer 4: 253-265, 2004.

53. Feng D: Screening and functional studies of miRNAs associated with drug-resistance and relapse in leukemia. Journal, 2011

54. Nam S, Long X, Kwon C, Kim S and Nephew KP: An integrative analysis of cellular contexts, miRNAs and mRNAs reveals network clusters associated with antiestrogen-resistant breast cancer cells. BMC Genomics 13: 732, 2012.

55. Liu MX, Siu MK, Liu SS, Yam JW, Ngan HY and Chan DW: Epigenetic silencing of microRNA-199b-5p is associated with acquired chemoresistance via activation of JAG1-Notch1 signaling in ovarian cancer. Oncotarget 5: 944-958, 2014.

This work is licensed under a Creative Commons Attribution-NonCommercial-NoDerivatives 4.0 International (CC BY-NC-ND 4.0) License. 